\title{
Mobile Barrier Deployment in Wireless Sensor Networks
}

\author{
Jun Liu ${ }^{1},{ }^{*}$ Lianglun Cheng ${ }^{1}$, Tao Wang ${ }^{1}$ and Jianhua Wang ${ }^{1}$ \\ ${ }^{1}$ School of Automation, Guangdong University of Technology, Guangzhou 510006 \\ China \\ liujunpaper@gmail.com llcheng@gdut.edu.cn 466586789@qq.com \\ 123chihua@163.com
}

\begin{abstract}
In wireless sensor networks (WSNs), barrier deploy is a hot topics. Barrier deploy for dynamic area is mobility and unsure. It is a important issue that sensors form an effective barrier. In this paper, we describe the problem of barrier deploy for dynamic area, define performance evaluation indexes of barrier deploy. A moved Barrier Deployment Algorithm based on swarm Intelligence of artificial fish swarm algorithm is developed. sensors can quickly find the border of the target area so as to form a closed barrier and response of the change of the target area. Through comparative simulations, we demonstrate that the proposed algorithm is effective for forming a barrier of dynamic area and it is little time delay and low energy cost.
\end{abstract}

Keywords: Mobile sensor network, Mobile Barrier; Deployment; Dynamic object; Convex Optimization

\section{Introduction}

Sensor networks as the underlying network of IoT (IoT: Internet of things) build a bridge for the physical world and information world. Sensor networks with many potential applications quickly become a hot topics. The barrier coverage has been attracting many scholars to study it [1-15]. A bulk of sensor nodes are organized as a sensing barrier for intrusion detection or target escaped, so called barrier deploy. Barrier deploy with a wide range of applications can be set on the border line to discover the stowaways, or be deployed around the safe to prevent thieves broke, or be deployed around contaminated area to prevent human and animal nearby.

Many efforts have been made for barrier deploy [5, 6, 14, 17] in the literature. But most of existing works focus on static objects such as the border line or critical areas whatever the mobile sensor node or the statics. However, in the presence of multiple applications of wireless sensor network, there are many objects need to be monitor are dynamic. The shape and position of objects are varying over time. In their scenarios, traditional methods of barrier coverage are failure for the aforementioned dynamic objects. It motivates us to study of mobile barrier deployment for dynamic objects in MSNs (MSNs: Mobile Sensor Networks).

Mobile barrier deployment for dynamic objects has application value in the practice. It can divide them into two categories: prevent internal object escape and external intrusion protection. Prevent internal object escaped, e.g., mobile barrier can outlines the area of crude oil spill in the ocean prevented pollutants diffusion freely, then sense and warn any entrant such as fish to leave away from this region. External intrusion 
protection, e.g., mobile barrier can keep moving surround a marching troop prevented external intrusion.

Due to characteristics of dynamic objects, conventional barrier deployment can't meet needs. The most important feature of mobile barrier deployment for dynamic is that mobile barrier transforms according to the change of dynamic objects. This feature brings about new issues for research. First, how do mobile sensor nodes recognize boundary of dynamic objects and forms a closed barrier. Second, how do mobile barrier quickly detects of boundary change and transforms according to dynamic boundary. Third, we also are interested in the issue that is to provide MBC while maximizing its monitoring performance and reducing the cost of sensor movement.

The main contributions and results of this work are summarized as follows:

1. The proposed algorithm achieves mobile barrier coverage for dynamic objects.

2. We given several evaluation index of mobile barrier.

3. Our algorithm is distributed, and every decision taken is based on local neighborhood information only.

4. It is efficient, since the basic idea of proposed algorithm is minimizes the length of barrier and maximizes the $\mathrm{K}$ value of mobile barrier.

The rest of this paper is organized as follows: The related work is presented in Section II. The problem is formulated in Section 3, and include state of the art, assumptions, definitions, and a problem statement. We proposed deployment algorithm in Section 4. Section 5 discussed our algorithm properties. We evaluated the performance of the proposed algorithm by simulations in Section 6. Section 7 presented the conclusion and discussed the future work.

\section{Related Work}

In recent years, barrier deployment is becoming a research focus of WSN. There are some researches were done. A variety of barrier deployments involving formation solutions for applications have been proposed in the literature. In [1], the issue of barrier coverage is firstly raised and an algorithm is proposed be used to judge whether the global barrier formed. Then, a distributed algorithm proposed in [2] is used to judge the local barrier formed. Reliable density estimation is studied in [3], and strong barrier coverage is presented in [4]. Methods of evaluated barrier deployment performance are studied in $[5,6]$ has studied barrier coverage with energy efficiency. These work mentioned above are about fixed barrier for static objects. To reduced the number of nodes for barrier deployment, a multi-round sensor deployment algorithm avoided deploy errors is proposed in [7]. For detected of one direction of crossing barrier, oneway barrier coverage is proposed in [8]. In some scenarios, there are not enough nodes to form a closed barrier. A multiple cooperative sensors patrol strategy is proposed in [9].

Recently, with the rapid development of MEMS, mobile sensor networks has become increasingly common in some applications. With mobile sensors arise, It will also generate interesting new research questions [10,11]. Optimal movement strategy for mobile sensor networks is studied in [12]. In this case of limited distance of sensors moved, a method of adjusted sensors location for high probability barriers is put forward. How do sensors move for barrier deployment in a scenario of nodes randomly distributed? This issue is studied in [14]. All of mention above is about mobile sensors barrier deployment for static objects. 
Nevertheless, there are monitoring objects with dynamic boundary and shifted position in the application of wireless sensor network such as pollutants on the sea, forest fires and move forces protection. For this type of problem, we defined as mobile barrier deployment of dynamic object. There is few work for this problem. To maximize value of $\mathrm{K}$, elastic barrier Algorithm is proposed in [15] for mobile barrier deployment of dynamic object. In this paper, our objective is quickly built a closed barrier for dynamic object and optimal performance of mobile barrier.

\section{Problem Formulation}

\section{A Problem statement}

Mobile barrier deployment is that mobile sensors self-organized build mobile barrier for area of interest monitored in the plane $\mathrm{M}$.

Definition 1. [Dynamic object: $O$ ] A dynamic object $O$ is an area with a continuous enclosed boundary, which deforms and moves on $\mathrm{M}$.

In this paper, we suppose that dynamic object is a simply connected region and it can autonomous move and transform without split. Mobile barrier built need to focus on boundary dynamic changed. The boundary of dynamic object denotes by L. The maximum movement speeds is denoted by $v_{0}$.

Definition 2. [Sensor node $S=\left\{s_{i}\right\}$ ]. There are $\mathrm{n}$ sensors in area, denoted by $\mathrm{S}=\{\mathrm{si}$, $\mathrm{i}=1, \cdots, \mathrm{n}\}$.

Sensors can move arbitrarily in the plane $\mathrm{M}$ with the maximum movement speed $V_{s}$. Sensors can recognize boundary of dynamic object within its sensing range and can detect intruders. In this paper, we adopt the widely used homogeneous disk model, where every sensor ${ }^{s_{i}}$ has an equal sensing rang $r_{s}$ and an equal communication range $r_{c}$.It assumed that $r_{c}$ is greater than $2 r_{s}$.

Definition 3. [Dynamic belt $(\lambda 1, \lambda 2, w)$ ]. It is defined as a closed belt region bounded by two closed curves $\lambda 1$ and $\lambda 2$, whose length are denoted by $l_{1}$ and $l_{2}$. The width of belt is $\mathrm{w}=\mathrm{d}(\lambda 1, \lambda 2)$.

B Evaluation Index

The first problem need to figure out is that evaluate belt deployment quality. In sensor network, our goal is quickly build a close barrier belt which quickly response with dynamic object. The evaluation index as follow:

Index 1.Time: T. It is defined as the time take out by sensors build a closed mobile barrier.

Index 2.Distance:D. It is defined as the sum of travel distances of all sensors in a time period T. $d_{i}$ is the distance travelled by sensor $s_{i}$ in time period T. The total travel distance is given by 


$$
D(0, T)=\sum_{i=1}^{n} d_{i}(0, T)
$$

Energy saving is crucial in the design of WSNs. For energy efficiency, it is important to reduce the total travel distance.

Index 3. Number of barriers: $K$. A dynamic belt has $\mathrm{K}$ barriers if and only if an intruder is detected by at least $\mathrm{K}$ sensors when it crosses by any path. Maximized K value can enhance a higher overall reliability of mobile barrier.

C Problem Model

Since the dynamic object may be any shape and the number of sensors is fixed, sensor should be deploy on the shortest barrier belt.

Theorem 1. The shortest barrier belt is convex hull of the dynamic object.

Proof: The definition of convex hull is that a set $\mathrm{D}$ of points in the Euclidean plane or Euclidean space is the smallest convex set that contains D.

It is supposed that the dynamic object is as a set D. According to the raised analysis theory[16], for any shape graphs D, the shortest closed curve is its convex hull.

Theorem 2. The maximum values of $\mathrm{K}$ is $\frac{2 n r_{s}}{l_{c}(A)}$.

Proof: From analysis above, the shortest closed barrier belt is convex hull of graph. The perimeter of convex hull of dynamic area $\mathrm{A}$ is denoted as $l_{c}(A)$.

Let's suppose that there are $\mathrm{n}$ sensors distributed randomly overall supervision area. The maximum length of barrier belt is $2 n r_{s}$ while all of nodes ranged along a straight line without overlapped. In this case, the distance of nodes is $2 r_{s}$. Therefore,

$$
K_{\max }=\frac{2 n r_{s}}{l_{c}(A)}
$$

In this paper, dynamic objects is a area with any shape, and can moved in any direction at rate $v_{0}$. For simplicity sake, suppose the shape of dynamic objects is a rectangle. It is by uniform motion in a certain direction. We research mobile barrier deployment where dynamic object covered by single-layer barrier under above hypothesis.

Figure 1 shows the scenario that the response of mobile barrier when object moving. Sensors at the edge A moved to right with boundary of dynamic object in real time. Sensors at the edge $C$ will lag behind dynamic object. Sensors at the edge B and D do not detect change of boundary and will not move. With dynamic object moved, gaps will appeared at junction between edge $\mathrm{A}$ and $\mathrm{B}$ or D. This gap is compensated by sensors on edge B and D moved toward right. How sensors move is the first thing we need to study. 


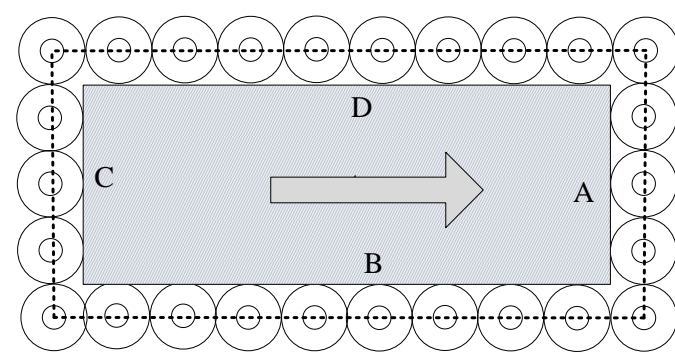

(a)

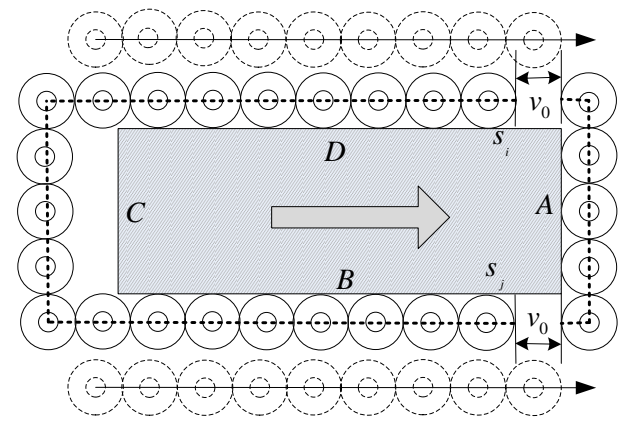

(b)

Figure 1. The response of mobile barrier with dynamic object moved

Sensors $S_{i}$ and ${ }^{S}$ at the gap send the message of gap to the following node while they detected the gap appear. Then all of sensors on edge B and D move to the right. There are $\mathrm{m}$ sensors on each edge $\mathrm{B}$ and $\mathrm{D}$. So total delay is:

$$
T=(m-1) T_{d}+\frac{v_{0}}{v_{s}}
$$

$T_{d}$ is once communication delay between sensors.

We would seem from (1), the length of barrier and communication delay have the most impact. However, if there are multilayer barrier on the boundary, redundancy sensors can quickly move to make up for the gap.

Form what we mention above, the max $\mathrm{K}$ is $\frac{2 n r_{s}}{l_{c}(A)}$. With the boundary changing, $\mathrm{K}$ probably is non-integer and that is means all of sensors would fail to just finish $\mathrm{n}$ mobile barrier. How to extra sensors distribute which is worth studying.

Two deployment modes are studied in [15]. In this paper, multilayer deployed is believed to waste $\frac{2 \pi m}{2 r_{s}}$ sensors, however, the value of $\mathrm{K}$ is greater with single denselydeployed. Single densely-deployed and multilayer deployed have the same effect when there are a lot of sensors. When $\mathrm{K}$ is non-integer, excess sensors may be randomly deployed in anywhere of boundary. In this case, once gap appeared, sensors do not make up for the gap in time while there are no sensors within easy reach. For single densely-deployed, once gap appeared, sensors distributed evenly can quickly complement the gap of barrier belt. Based on the analysis and conclusion in front, single densely-deployed is more appropriate to barrier deployment of dynamic object.

\section{Barrier Deployment Algorithm}

The analysis in the previous section provides us with conditions need to be fulfilled for optimized barrier deployment. In this section, we analyze what strategy should be adopted to achieve mobile barrier deployment. 


\subsection{Idea of Barrier Deployment}

There are two steps to achieve barrier deployment for dynamic object: boundary recognized and barrier build.

Step 1: boundary recognized

Because of dynamic of object sharp, the first thing for barrier deployment is boundary seeking. In this step, we considerate to absorb idea of AFSA(Artificial fish swarm algorithm, AFSA) [17]. AFSA is a new evolution algorithm based on swarm intelligence simulating social behavior of fish schooling. There are three types of typically behavior: Prey, Follow, Swarm. In the boundary seeking process, the process of sensors approaching boundary is the equal of the process of artificial fish search of food.

Sensor ${ }_{j}$ performs the following steps according to self-state and neighbor information:

Firstly, sensor detect that is not at the boundary. Sensors perform behavior of prey which randomly moved to a new position with a maximum step length ${ }_{j}$ meanwhile maintain network connected.

$$
P_{j}^{\prime}=P_{j}+\operatorname{rand}\left(l_{j}\right) P
$$

The number of $\operatorname{rand}\left(l_{j}\right)$ is a randomly number between 0 and $l_{j}$. The vector $P$ of the unit vector means that sensors moved to any direction. Whenever sensors move to a new position, they communication with neighbors and detect boundary information. If boundary is found, it is means prey success, otherwise prey continuing.

If there is any boundary information round them or their neighbors, sensors carry on follow behavior. Sensor $S^{*}$ denoted the neighbor node whose have boundary information.

$$
\begin{gathered}
P_{j}^{\prime}=P_{j}+\tilde{l}_{j} \frac{\left(P^{*}-P_{j}\right)}{d\left(s_{j}, s^{*}\right)} \\
\tilde{l}_{j}= \begin{cases}\frac{1}{2} d\left(s_{j}, s^{*}\right), & \frac{1}{2} d\left(s_{j}, s^{*}\right)<l_{j} \\
l_{j} & \text { or }\end{cases}
\end{gathered}
$$

$P^{*}$ is the position of $S^{*}$.

Step 2: barrier build

Sensors move to boundary of dynamic object when found boundary and deploy along the edge of boundary. Node at both ends move forward that sensors getting together stretched as a chain. Each expand of chains is by sensors joined and this information is propagated to the entire chain. 


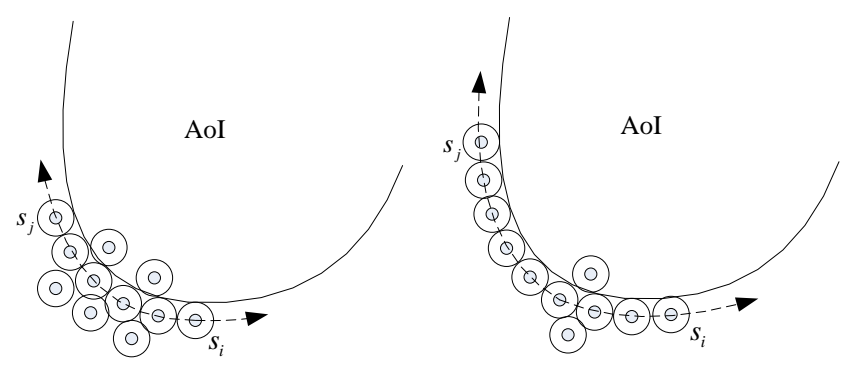

Figure 2. Mobile sensors distributed of chain barrier build

With chains extended, different chains combine into a long chain. During chains building, barrier belt do not complete until one chain linked end-to-end.

Disposed of Boundary of Inward Concave

From previous section analysis, sensors should deploy on convex hull of dynamic object that maximize K. Barrier deployment of inward concave adopt geometric vector method.

$$
F=F_{s_{i} s_{i-1}} \square F_{s_{i} s_{i+1}}
$$

$F_{s_{i} s_{i-1}}$ and $F_{s_{i} s_{i+1}}$ are vectors of sensor ${ }^{s_{i}}$ pointed at neighboring nodes respectively. If $\mathrm{F}$ is positive values under the hypothesis of boundary tangent as horizontal line, sensor

$s_{i}$ move outwards, else without move.

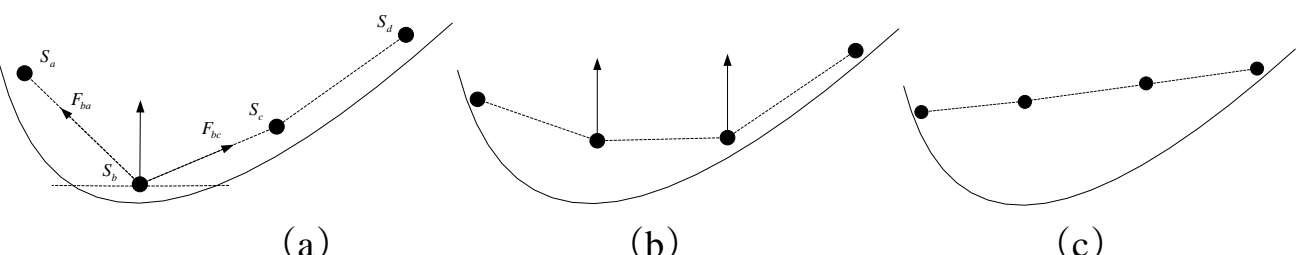

Figure 3. Barrier belt build of inward concave

\subsection{Algorithmic Design}

According to analysis above, we proposed moved barrier deployment algorithm (MBDA: Mobile Barrier Deployment Algorithm). The algorithm can achieved barrier coverage of any dynamic object by adjusted mobile sensors position.

The detailed algorithm is shown as follows:

\section{Algorithm 1(MBDA: Mobile Barrier Deployment Algorithm)}

Input: the sensing range $r_{s}$, the communication range $r_{c}$.

Output: Boundary formation

//Step 1: Boundary Recognition

repeat 


\section{If (Si detected barrier information) THEN}

$\mathrm{Pi} \leftarrow$ (5)

\section{ELSE}

$\mathrm{Pi} \leftarrow(4)$

\section{END}

//Step 2 : Barrier Construction

Switch (Num of immediate neighbors)

Case 2:

If $F=F_{s_{i} s_{i-1}} \square F_{s_{i} s_{i+1}}>\mathbf{0}$

Pi moved out.

\section{ELSE}

Pi moved ahead.

Case 1:Pi moved ahead.

break;

Default : Pi moved ahead either sides.

break;

\section{Until}

$$
s_{i+1}=s_{j} s_{j+1}=s_{i}
$$

The algorithm runs on each sensor node which randomly distribute on monitoring area. During algorithm running, nodes seek boundary of object. Once boundary found, nodes broadcast to the surrounding area, and neighboring nodes quickly are drew to there by proposed strategies above. There are several barrier chains distributed on boundary of object. Sensors on two ends of each barrier chain move forward. Different barrier chains merged together when they meet. Barrier belt do not formed until the first node and the last node of some barrier chain meet.

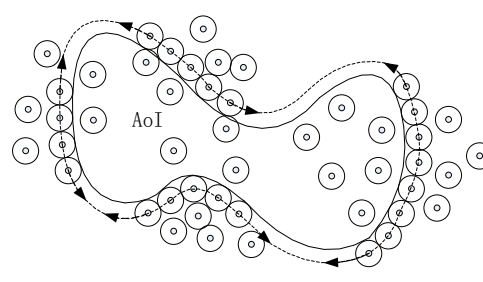

(a) Boundary recognized

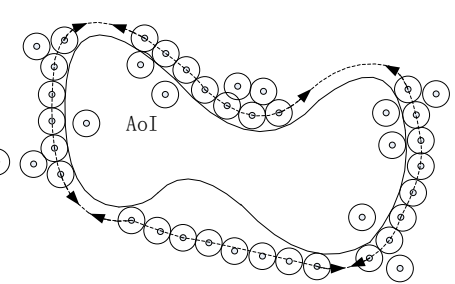

(b) Barrier deployment of Boundary of Inward Concave Closed barrier formed

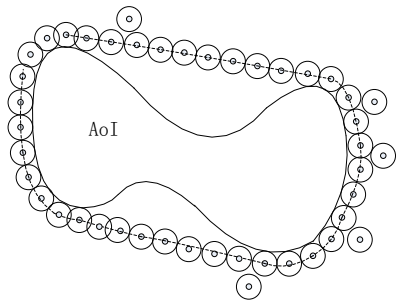

(c) Concave

Figure 4. Three snapshots of the mobile barrier formation process 


\subsection{Algorithmic Analysis}

Theorem 3: Barrier belt do not formed until the first node and the last node of some barrier chain meet.

Proof: (Absurdum) Let us suppose there are $\mathrm{n}$ barriers $l_{1}, l_{2}, \ldots l_{n}$ distributed on boundary of dynamic object. $s_{i}$ and ${ }^{s_{j}}$ respectively are the first node and last node of barrier $l_{k}$.

If $s_{i+1}=s_{j}$ or $s_{j+1}=s_{i}$ during the barrier $l_{k}$ expanded on both ends, barrier belt of dynamic object build is unfinished.

The barrier $l_{k}$ is a closed barrier belt because of $s_{i+1}=s_{j}$ or $s_{j+1}=s_{i}$. And because of barrier belt of dynamic object unfinished, the area of the barrier $l_{k}$ surrounded cannot be object area.

We can draw a conclusion that $l_{k}$ is not on the boundary of object.

This conclusion conflicts with the prior declarations that barriers $l_{1}, l_{2}, \ldots l_{n}$ are distributed on convex hull of object area.

Therefore, theorem 3 is created.

\section{Performance Evaluation}

In this section, we evaluate the performance of the algorithms by simulation experiment.

\subsection{Emulation Environment}

There are three scenarios for simulation experiment. It assume that 50 sensors are randomly distributed in one area of $400 * 600 \mathrm{~m}$. In Figures 5, 6, 7, the gray area represents the dynamic object. We suppose that a sensor can detect and distinguish dynamic object area within its sensing range $s_{i}=25 \mathrm{~m}$. Communication ranges of sensors is $S_{c}=50 \mathrm{~m}$. The velocity of a sensor is at most $10 \mathrm{~m} / \mathrm{s}$. Test scenes divides into static areas and dynamic areas. Test indicators include spending time of build barrier and distance of total sensors moved.

\subsection{Performance Analysis}

Figures 5, 6, 7 show snapshots of mobile barrier deployment process for different objects respectively. We can see that all of scenes are formed closed barrier belts. The simulation results have verified the validity and practicability of the model and algorithm. Figure 7 shows the scene of object moved to one direction. The mobile barrier could response the dynamic object in time. 


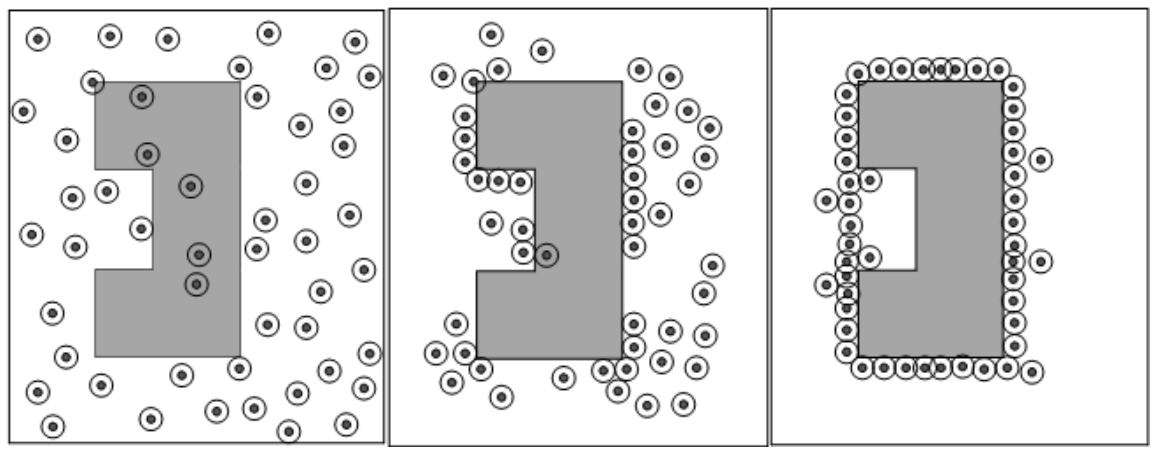

(a)

(b)

(c)

Figure 5. Three snapshots of the mobile barrier deployment process for the static object in regular pattern simulation

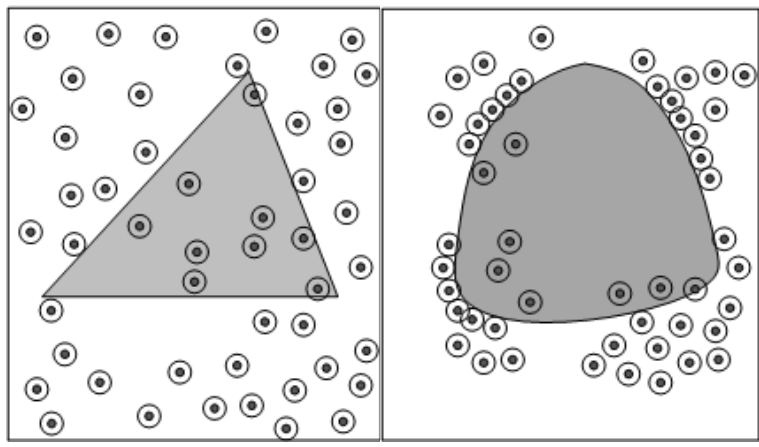

(a)

(b)

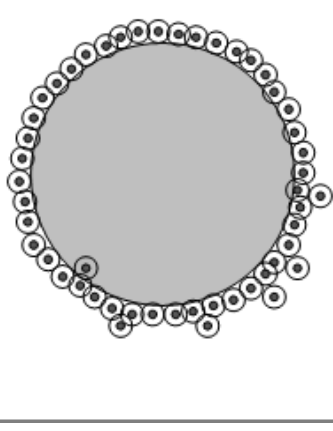

(c)

Figure 6. Three snapshots of the mobile barrier deployment process for the dynamic object simulation

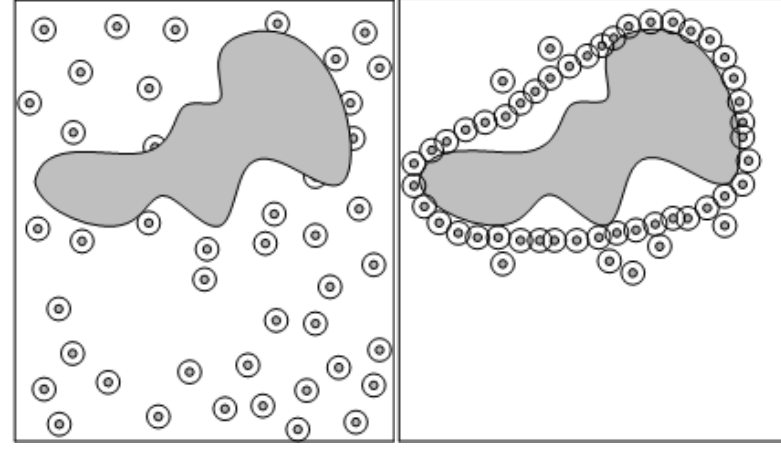

(a)

(b)

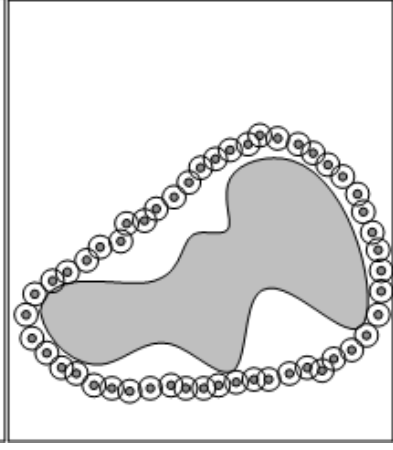

(c)

Figure 7. Three snapshots of the mobile barrier deployment process for the moving dynamic object simulation

In our test environment, we compared performance in proposed indicators above between EBA proposed in [15] and our proposed MBDA. The distance of total sensor nodes moved each run two algorithms are showed in Fig.8. The accumulated travel distance in three scenes for EBA over time 600 s is $57216 \mathrm{~m}, 45832 \mathrm{~m}$ and $28251 \mathrm{~m}$ 
respectively. The accumulated travel distance for MBDA is $45272 \mathrm{~m}, 32932 \mathrm{~m}, 18253 \mathrm{~m}$ which is less than EBA. The duration time the barrier formation process is completed is $563 \mathrm{~s}, 492 \mathrm{~s}$ and $417 \mathrm{~s}$ respectively for EBA. That is greater than MBDA which is $513 \mathrm{~s}, 428 \mathrm{~s}, 367 \mathrm{~s}$. This denoted that our proposed algorithm could quickly achieved barrier deployment which has benefited from the strategy of sensors moved.

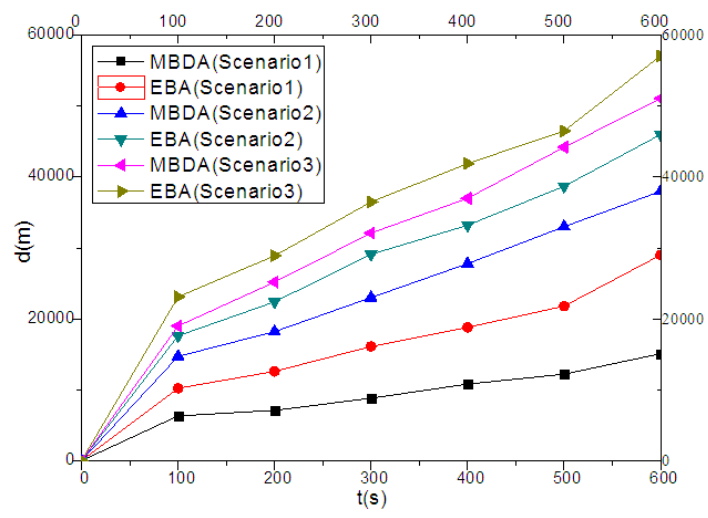

Figure 8. The accumulated travelled distance of all sensors over time

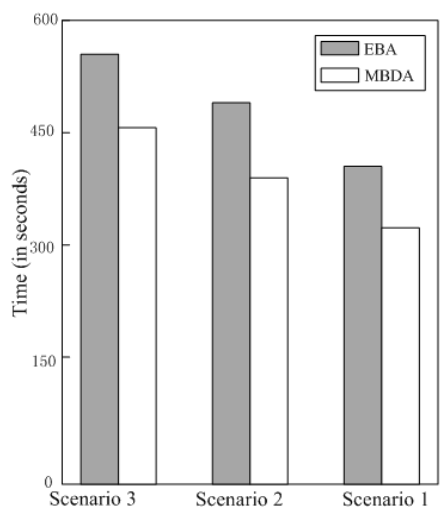

Figure 9. The convergence duration in three scenarios

\section{Conclusion}

In this paper, the problem of mobile barrier deployment for dynamic object is presented. The mobile barrier deployment algorithm is proposed on sensors to move cooperatively and form maximal $\mathrm{K}$ strong barrier. This algorithm could quickly response the change of dynamic object. The method of sensors seek boundary borrows from the idea of artificial fish swarm algorithm that could form a closed barrier belt quickly.

Since the model of mobile barrier deployment for dynamic object is difficult to accurately evolve, there exist several open questions for further study. One of the future works is specific condition of sensors speed of movement for different movement and change modes of dynamic object. Besides, energy efficiency is critical for mobile sensors. The optimal moved path is another future work. 


\section{Acknowledgements}

This work was supported in part by Key project of national natural science foundation of China united with Guangdong mutual foundation (U1201251), Foundation for Distinguished Young Talents in Higher Education of Guangdong (LYM11057), the Scientific Research Project of Guangzhou City (12C42111582) and Doctor Start Project of Natural Science Foundation of Guangdong Province (S2012040006666).

\section{References}

[1] S. Kumar, T. Lai and A. Arora, "Barrier coverage with wireless sensors", Proceedings of the 11th Annual International Conference on Mobile computing and networking, ACM, (2005), pp. 284-298.

[2] A. Chen, S. Kumar and T. Lai, "Designing localized algorithms for barrier coverage", Proceedings of the 13th annual ACM international conference on Mobile computing and networking, ACM, (2007), pp. 63-74.

[3] P. Balister, B. Bollobas and A. Sarkar, "Reliable density estimates for coverage and connectivity in thin strips of finite length", Proceedings of the 13th annual ACM international conference on Mobile computing and networking, ACM, (2007), pp. 75-86.

[4] B. Liu, O. Dousse and J. Wang, "Strong barrier coverage of wireless sensor networks", Proceedings of the 9th ACM international symposium on Mobile ad hoc networking and computing, ACM, (2008), pp. 411-420.

[5] A. Chen, T. Lai and D. Xuan, "Measuring and guaranteeing quality of barrier coverage in wireless sensor networks", Proceedings of the 9th ACM international symposium on Mobile ad hoc networking and computing, ACM, (2008), pp. 421-430.

[6] J. Li, J. Chen and T. Lai, "Energy-efficient intrusion detection with a barrier of probabilistic sensors", INFOCOM, 2012 Proceedings IEEE, (2012), pp. 118-126.

[7] G. Yang and D. Qiao, "Multi-round sensor deployment for guaranteed barrier coverage", INFOCOM, 2010 Proceedings,IEEE, (2010), pp. 1-9.

[8] A. Chen, Z. Li and T. Lai, "One-way barrier coverage with wireless sensors", INFOCOM, 2011 Proceedings IEEE, (2011), pp. 626-630.

[9] S. He, J. Chen and X. Li, "Cost-effective barrier coverage by mobile sensor networks", INFOCOM, 2012 Proceedings, IEEE, (2012), pp. 819-827.

[10] W. Chen, J. Hou and L. Sha, "Dynamic clustering for acoustic target tracking in wireless sensor networks", Mobile Computing, IEEE, Transactions on, vol. 3, no. 3, (2004), pp. 258-271.

[11] B. Liu, P. Brass and O. Dousse, "Mobility improves coverage of sensor networks", Proceedings of the 6th ACM international symposium on Mobile ad hoc networking and computing, ACM, (2005), pp. 300-308.

[12] B. Bhattacharya, B. Burmester and Y. Hu, "Optimal movement of mobile sensors for barrier coverage of a planar region”, Combinatorial Optimization and Applications, (2008), pp. 103-115.

[13] A. Saipulla, C. Westphal and B. Liu, "Barrier coverage of line-based deployed wireless sensor networks", INFOCOM 2009, IEEE, (2009), pp. 127-135.

[14] L. Kong, X. Liu and Z. Li, "Automatic barrier coverage formation with mobile sensor networks", Communications (ICC), 2010 IEEE, International Conference on, IEEE, (2010), pp. 1-5.

[15] L. Kong, Y. Zhu, M. -Y. Wu and W. Shu, "Mobile Barrier Coverage for Dynamic Objects in Wireless Sensor Networks", IEEE MASS, Las Vegas, USA, (2012).

[16] D. P. Bertsekas, A. Nedić and A. E. Ozdaglar, "Convex analysis and optimization", Belmont, Athena Scientific, (2003).

[17] S. Wei, "Forecasting stock indices using radial basis function neural networks optimized by artificial fish swarm algorithm”, Knowledge-Based Systems, vol. 24, no. 3, (2011), pp. 378-385. 


\section{Authors}

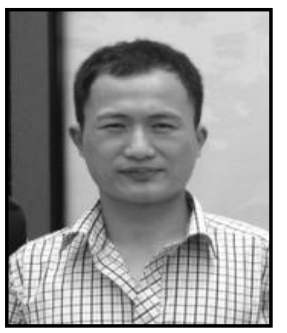

\section{Jun Liu}

Jun Liu received the B.E degree in Automation from Guangdong University of technology, China, in 2009. He is currently pursuing the Ph.D. degree in Guangdong University of technology, China. His previous research interests are in the area of mobile sensor networks, Thing of Internet, and Ad hoc network, with emphasis on sensors deployment.

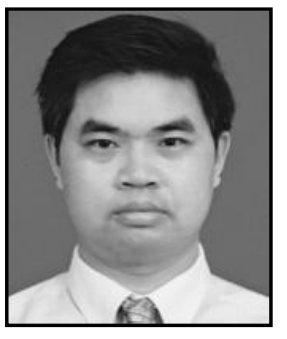

\section{LiangLun Cheng}

He was born on August 22, 1964 in Hubei. He received his M.S and Ph.D degrees from Huazhong University of Science and Technology, Hubei, China in 1992 and Chinese academy of Sciences Jilin, china in 1999 respectively. He is a Prof and doctoral supervisor of Guangdong University of Technology. His research interests include RFID and WSN, IoT and CPS, production equipment and automation of the production process, embedded system, the complex system modeling and its optimization control, software of automation and information, etc

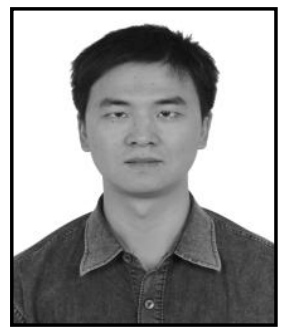

\section{Tao Wang}

He received the PHD Degree in network security from Sun Yat-Sen University, Guangzhou, China, in 2010. His current research interest includes context-aware computing, service composition, protocol optimization in wireless sensor network and cyber-physical system.

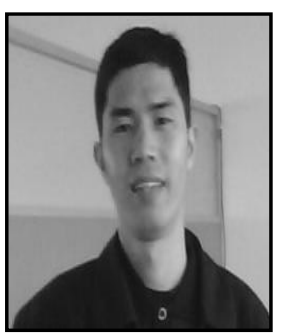

\section{JianHua Wang}

He was born on February 6, 1982 in Guangdong, China. He receivedhis B.S degree in Electronic Information Science and Technology from Shaoguan University, Guangdong, China, in 2006. Currently he is pursuing Ph.D degree in Control Science and Engineering at Guangdong University of Technology. His research interests include $3 \mathrm{G}$ wireless video transmission, cyber-physical systems, IoT and wireless sensor networks. 
International Journal of Control and Automation Vol.7, No.5 (2014) 